\title{
Fluoroscopic analysis of lumbar epidural contrast spread after retrograde interlaminar ventral epidural injection (RIVEI)
}

\author{
Ji Seon Jeong, Jae Chol Shim, Jung Pil Woo, and Jae Hang Shim \\ Department of Anesthesiology and Pain Medicine, School of Medicine, Hanyang University, Seoul, Korea
}

Background: Retrograde interlaminar ventral epidural injection (RIVEI) may hypothetically be more effective if the catheter is placed at the ventrocaudal aspect of the exiting nerve. We tested that hypothesis by measuring ventral and dorsal epidural contrast flow during RIVEI.

Methods: To perform RIVEI, a 17 G Tuohy needle was inserted to access the epidural space. A 19 G epidural catheter was inserted and advanced through the needle, passing in a caudal direction to the lower aspect of the contralateral pedicle. Fluoroscopic images were recorded at $1.5 \mathrm{ml}$ increments of contrast. Based on the images of contrast dispersal, the extent of contrast spreading was assessed in 82 patients.

Results: All 82 patients (100\%) injected with $3.0 \mathrm{ml}$ contrast medium demonstrated ventral epidural spreading. Mean spreading level from the catheter tip was $2.21 \pm 0.93$ with $3.0 \mathrm{ml}$ of contrast. Spreading to the superior aspect of the supra-adjacent intervertebral disc was observed in 67/82 (81.7\%) of RIVEIs with $3.0 \mathrm{ml}$ of contrast injected into the ventral epidural space. We found that $3.0 \mathrm{ml}$ of contrast reached the inferior aspect of the infra-adjacent intervertebral disc in 95.1\% (78/82) of RIVEIs performed.

Conclusions: Our findings imply that a one-level RIVEI may be sufficient in situations where a two-level injection would currently be used. (Korean J Anesthesiol 2013; 65: 431-437)

Key Words: Contrast media, Epidural injections.

Received: February 20, 2013. Revised: 1st, March 26, 2013; 2nd, April 23, 2013. Accepted: May 3, 2013.

Corresponding author: Jae Chol Shim, M.D., Ph.D., Department of Anesthesiology and Pain Medicine, School of Medicine, Hanyang University, Hanyang University Hospital, 17, Haengdang-dong, Seongdong-gu, Seoul 133-792, Korea. Tel: 82-2-2290-8680, Fax: 82-2-2299-8692, E-mail: jcshim@hanyang.ac.kr

(c) This is an open-access article distributed under the terms of the Creative Commons Attribution Non-Commercial License (http:// creativecommons.org/licenses/by-nc/3.0/), which permits unrestricted non-commercial use, distribution, and reproduction in any medium, provided the original work is properly cited. 


\section{Introduction}

In disc herniation, as in spinal stenosis, optimal fluoroscopic imaging requires the accurate and complete delivery of contrast medium by needle into the central spinal segment where the lesion is located. Transforaminal (TF) approaches for injection are technically uncomplicated. But although the radiologic target advocated for transforaminal injections corresponds to the superoanterior region of the foramen, needle placement anterior to the "safe triangle" region may be complicated if the foramen is distorted by stenosis, and in far lateral disc herniations, spondylolisthesis and scoliosis. In these situations, the spinal nerve location in the intervertebral foramen is elevated, and the needle must avoid the anterior position [1].

The injection approach must therefore be adapted for each patient so as to achieve optimal delivery of drug or reagent to the site of pathology. While noble techniques [2-6] for needle insertion into the epidural space are under investigation, the welltolerated, relatively safe and effective procedure of transforaminal injection should be further explored as well. The retrodiscal zone has been described by Kambin: Kambin triangle. This is a right triangle overlying the dorsolateral disc $[7,8]$.

The inferior aspect of the Kambin triangle is defined inferiorly by the proximal vertebral plate, posteriorly by the articular process of the lower segment, and anteriorly by the exiting nerve root. In the Kambin triangle technique, steroids are instilled directly at the nerve-disc interface. The "safe triangle" transforaminal epidural steroid injection (TFESI) delivers steroids distal to the nerve disc interface, rather than directly [9]. Since epidural injection by the "safe triangle" approach is made at the ventrocranial aspect of the exiting nerve, we hypothesized that for the ventral epidural injection, the catheter may be placed ventrocaudal to the exiting nerve, that is, along the hypotenuse of the Kambin triangle. We refer to this approach as retrograde interlaminar ventral epidural injection (RIVEI)

We are interested in determining whether medications administered by this RIVEI approach will spread into the ventral epidural space as described for the TFESI. Of particular interest is the extent to which local anesthetics or contrast media spread into the ventral and dorsal epidural spaces in patients who suffer from radiating pain due to spinal stenosis and disc herniation. This study was designed to evaluate these topics using fluoroscopically guided RIVEI.

\section{Materials and Methods}

The Hanyang University Hospital Institutional Review Board approved this study protocol, and each participant gave written informed consent. Eighty-seven patients were enrolled who underwent RIVEI at our pain clinic and met the following inclu- sion criteria: (1) presence of radiating lumbar pain and (2) clear evidence of nerve root compression with either disc herniation or central canal and/or lateral recess stenosis based on clinical and cross-sectional imaging studies (computed tomography or magnetic resonance imaging). Exclusion criteria included age younger than 18 years, allergy to contrast media, inability to consent, prior surgery at the lumbar level, and presence of contraindication to epidural catheter placement (coagulopathy, infection at the site of insertion, or septicemia).

Each patient was placed in a prone position, prepared with topical antiseptics at the injection site, and draped in sterile cloth. The corresponding author was present for all procedures. Under fluoroscopic guidance using a cranio-oblique tilt to obtain a single contour of the upper endplate of the corresponding vertebra, the interlaminar space between the vertebrae with disc pathology was identified in an antero-posterior (AP) projection with the patient prone. A line connecting the lower portion of the pedicle where the corresponding symptomatic spinal nerve exits at the level below the disc herniation and the point corresponding to the contralateral medial-most part of the interlaminar space was drawn. Along that line, the skin entry point wheal was penetrated contralateral to the level superior to the interlaminar space with $1 \%$ lidocaine. Local anesthetic was infiltrated down to the lamina along that line. A 17-gauge Tuohy needle was inserted at the entry point and advanced in a medial direction until penetration of the epidural space in the midline between the appropriate lamina was achieved using the loss-ofresistance technique. If bony contact was made at the medialmost part of the contralateral interlaminar opening, the tip of the needle was redirected caudomedially and inserted into the epidural space.

Once placed at the epidural space, a 19-gauge radio-opaque spring-tipped epidural catheter with a wire stylet (Theracath ${ }^{\circledR}$; Arrow International, PA, USA) was passed in a caudal direction into the epidural space [10]. If resistance was met, the catheter was removed and the needle placement rechecked and readjusted if necessary (The catheter should advance easily, and should never be forced). Using the curvature of the Tuohy needle opening to direct the catheter tip, the catheter was steered out to the lower aspect of the pedicle into the ventral epidural space and the neural foramen until the catheter tip was located below the bisection of the pedicle. AP and lateral spot radiographs were obtained to confirm the position of the catheter and tip. Contrast medium was then injected in two $1.5 \mathrm{ml}$ portions under real-time continuous fluoroscopy at a consistent rate of 0.5 to $1.0 \mathrm{ml} / \mathrm{min}$, as monitored manually [11]. Real-time continuous imaging was used to assess the pattern of contrast dispersal into the ventral and dorsal epidural spaces, as well as to monitor for intravascular injection. Fluoroscopic AP and lateral spot radiographic images were recorded as $1.5 \mathrm{ml}$ increments of contrast 

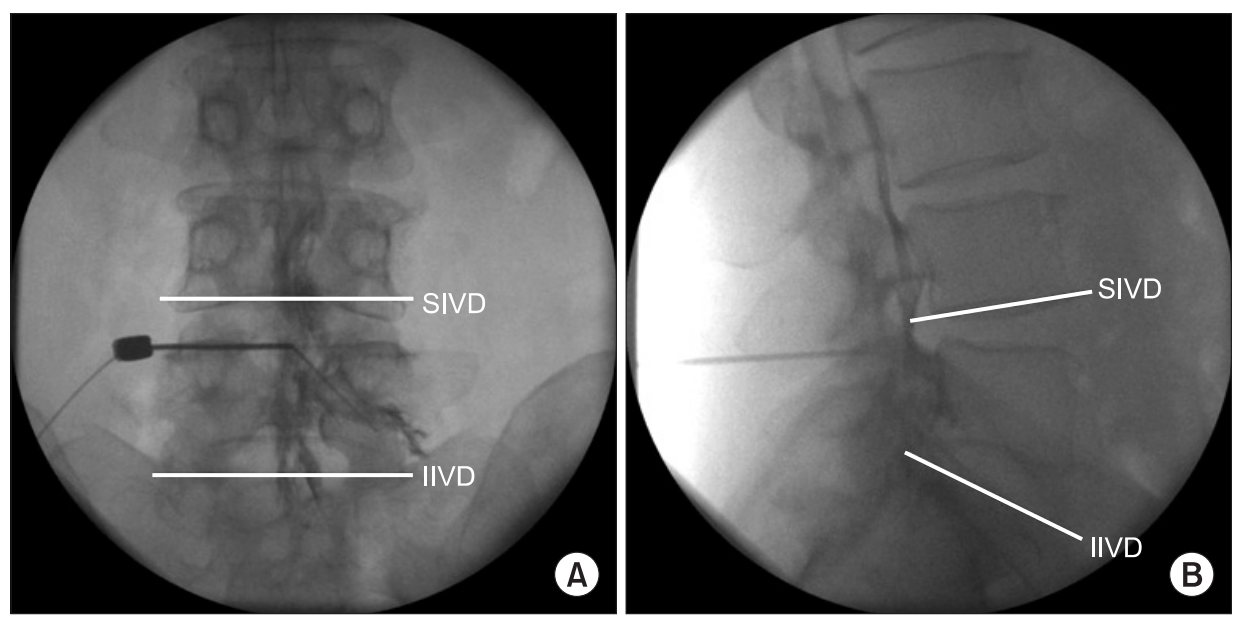

Fig. 1. Contrast spread into the lumbar epidural space following RIVEI. (A) Anteroposterior view of a catheter placed for a right L5 RIVEI demonstrating the landmarks used for this investigation. Note the SIVD and IIVD. Also, note the contrast flowing along the most medial aspect of the superior pedicle. (B) Lateral view of a catheter placed for a right L5 RIVEI demonstrating the landmark used for this investigation. Note the SIVD and IIVD. SIVD: superior aspect of the supraadjacent intervertebral disc, IIVD: inferior aspect of the infra-adjacent intervertebral disc.

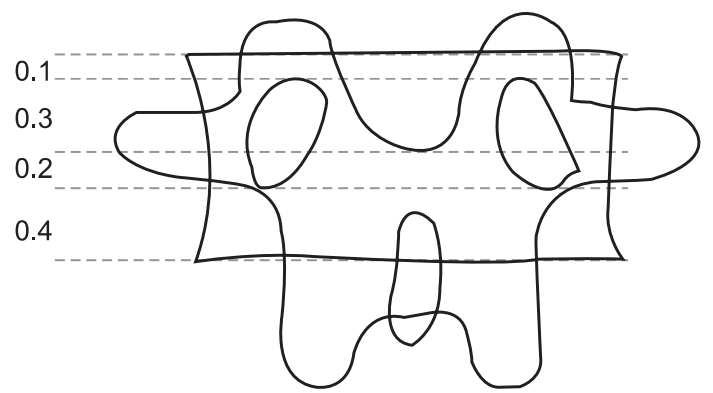

Fig. 2. Schematic representation of a lumbar vertebra shows the height of the interlaminar space relative to the pedicle and vertebral body.

were injected (Fig. 1).

The ventral and dorsal flow patterns from the catheter tip were further described as being cephalad or caudad. Catheter tip position and the extent of contrast spreading to the cephalad and caudal directions from the catheter tip were recorded using the chart of a typical lumbar vertebra showing the height of the interlaminar space relative to the pedicle and vertebral body [12] (Fig. 2). Spread of contrast upward to the superior aspect of the supra-adjacent intervertebral disc (SIVD) and to the inferior aspect of the infra-adjacent intervertebral disc (IIVD) was evaluated by viewing the lateral projection fluoroscopic images (Fig. 1). To evaluate the potential therapeutic coverage of the superior and inferior discs, we used the SIVD and the IIVD targets, respectively [11].

Ventral spread was evaluated by the spread of contrast to the level of the posterior longitudinal ligament or abutting the posterior aspect of the contigious vertebral body(s) at the level of the catheter tip [2]. If the contrast did not fill in one-third of the ventral area of the spinal canal, the filling was considered dorsal [13]. If a fluoroscopically confirmed vascular injection was noted, the patient's data was excluded from this analysis since we were unable to quantify their contrast flow.
The number of lumbar intervertebral levels of flow was recorded. A level of flow was defined by the relative positions of the interlaminar space and pedicle and the vertebral body [12] (Fig. 2). The ratios of pedicle height and interlaminar space to the vertebral body height were defined as 0.5 and 0.4 , respectively. The ratio of the distance from the lower border of the pedicle to the caudal edge of the interlaminar space to the vertebral body was defined as 0.2 . The ratio of the distance from the lower border of the pedicle to the lower endplate to the vertebral body was defined as 0.4 . The ratio of the distance from the margin of the upper endplate to the upper border of the pedicle was defined as 0.1 .

The paired t-test was used to compare continuous variables. The McNemar test was used to test differences in proportions. A $\mathrm{P}<0.05$ was considered significant. Statistical calculations were performed using STATA ver. 11.0 (StataCorp, College Station, Texas, USA).

\section{Results}

Two patients were excluded from the analysis because the interventionalist failed to advance the catheter into the intervertebral foramen at the level of the pedicle after successfully placing the catheter into the dorsal epidural space. Three patients were excluded from the analysis because the contrast injection was intravascular. The data from 82 patients were included in this analysis (Tables 1 and 2).

Ventral epidural spreading was demonstrated in all patients ( 82 of 82 ) given $3.0 \mathrm{ml}$ contrast and in 81 of 82 patients given 1.5 $\mathrm{ml}$. Both ventral and dorsal epidural spreading were observed in 39 of $82(47.6 \%)$ patients injected with $3.0 \mathrm{ml}$ of contrast. Only ventral epidural spread was observed in 43 of 82 (52.4\%) patients injected with $3.0 \mathrm{ml}$ of contrast (Table 3). The demonstration of both ventral and dorsal epidural spreading of contrast differed significantly between patients given contrast volumes of 
$1.5 \mathrm{ml}$ and $3.0 \mathrm{ml}(\mathrm{P}<0.05)$ (Table 3$)$.

The ventral epidural spreading levels (mean $\pm \mathrm{SD}$ ) from the catheter tip were $1.60 \pm 0.71$ and $2.21 \pm 0.93$ in patients injected with 1.5 and $3.0 \mathrm{ml}$ of contrast, respectively (Table 4 ). The difference between the mean spreading levels following injection of 1.5 $\mathrm{ml}$ and $3.0 \mathrm{ml}$ of contrast was significant $(\mathrm{P}<0.05)$.

After injections of 1.5 and $3.0 \mathrm{ml}$ of contrast, 59 of 82 (71.9\%) RIVEI and 67 of 82 (81.7\%) RIVEI, respectively, showed spreading into the SIVD in the ventral epidural space (Table 5). We

Table 1. Demographic Data of 82 Patients with Radiating Lumbar Pain and Nerve Root Compression

\begin{tabular}{cccccc}
\hline $\begin{array}{c}\text { Target level } \\
(\mathrm{L} 4 / \mathrm{L} 5)\end{array}$ & $\begin{array}{c}\text { Laterality } \\
(\mathrm{Rt} / \mathrm{Lt})\end{array}$ & $\begin{array}{c}\text { Age } \\
(\mathrm{yr})\end{array}$ & $\begin{array}{c}\text { Gender } \\
(\mathrm{M} / \mathrm{F})\end{array}$ & $\begin{array}{c}\text { Weight } \\
(\mathrm{kg})\end{array}$ & $\begin{array}{c}\text { Height } \\
(\mathrm{cm})\end{array}$ \\
\hline $14 / 68$ & $35 / 47$ & $56.2 \pm 13.7$ & $22 / 60$ & $61.6 \pm 9.3$ & $159.7 \pm 8.6$ \\
\hline
\end{tabular}

Values are number of patients or mean \pm SD. Rt: right side, Lt: left side, M: male, F: female.

Table 2. Spinal Conditions Evaluated for Contrast Spread during RIVEI

\begin{tabular}{lc}
\hline \multicolumn{1}{c}{ Diagnosis } & $\begin{array}{c}\text { Number of } \\
\text { patients }(\%)\end{array}$ \\
\hline Lumbar disc herniation & $37(45.1)$ \\
Central spinal stenosis & $19(23.2)$ \\
Both lumbar disc herniation and central stenosis & $21(25.6)$ \\
Foraminal stenosis & $5(6.1)$ \\
Total & 82 \\
\hline
\end{tabular}

RIVEI: retrograde interlaminar ventral epidural injection.

Table 3. Patterns of Contrast Flow into Epidural Space following RIVEI

\begin{tabular}{lcc}
\hline \multirow{2}{*}{\multicolumn{1}{c}{ Epidural spreading }} & \multicolumn{2}{c}{ Injected volume of contrast } \\
\cline { 2 - 3 } & $1.5 \mathrm{ml}$ & $3.0 \mathrm{ml}$ \\
\hline Ventral epidural & $81 / 82(98.8)$ & $82 / 82$ \\
Dorsal epidural & $34 / 82(41.5)$ & $39 / 82(47.6)$ \\
Both (ventral and dorsal) epidural & $33 / 82(40.2)^{*}$ & $39 / 82(47.6)^{*}$ \\
Ventral epidural only & $48 / 82(58.5)$ & $43 / 82(52.4)$ \\
Dorsal epidural only & $1 / 82(1.2)$ & $0 / 82$ \\
None & 0 & 0
\end{tabular}

Values are number of patients (\%). RIVEI: retrograde interlaminar ventral epidural injection. ${ }^{*} \mathrm{P}<0.05 ; 1.5 \mathrm{ml}$ vs $3.0 \mathrm{ml}$ with $\mathrm{McNemar}$ test. found that in 78 of 82 patients (95.1\%), a $3.0 \mathrm{ml}$ injection of contrast reached the IIVD in the ventral epidural space (Table 5). The rate of contrast spreading to the SIVD in the ventral epidural space differed significantly for injections of $1.5 \mathrm{ml}$ and $3.0 \mathrm{ml}$ $(\mathrm{P}<0.05)$. Catheter tip localization levels (mean $\pm \mathrm{SD})$ from the lower endplate were $0.14 \pm 0.15$ and $0.15 \pm 0.14$ at L4 and L5, respectively. No patient developed any adverse reaction to contrast media or adjuvant medications.

\section{Discussion}

TFESI provides palliative relief to patients with a variety of spinal conditions, and may also be applied diagnostically. Placement of the needle tip may be critical to the success of this procedure, as the catheter threaded through the needle guides the medicine or contrast material to the lesion site. Needle placement may be impeded, however, in disc conditions such as upward migration, far lateral herniations, foraminal abnormalities (spondylolisthesis, spinal stenosis) and previous surgery. Moreover, proximity to the targeted nerve within the superior neural foramen during TFESI brings the needle close to the great anterior radiculomedullary artery, which presents a risk for vascular injury resulting in spinal cord ischemia and paraplegia [14-18].

Concerns regarding the safety and effectiveness of TFESI have focused attention on alternative injection routes, including lateral parasagittal interlaminar (PIL) [2], inferior foraminal (targeting the inferior aspect of the foramen) [6], retrodiscal $[4,5]$, and lumbar interlaminar ventral epidural (LIVE) approaches [3]. Candido et al. [2] found the PIL approach to be

Table 5. Spread of Contrast to Superior Aspect of the SIVD and Inferior Aspect of the IIVD following RIVEI

\begin{tabular}{|c|c|c|c|c|}
\hline & \multicolumn{2}{|c|}{ Ventral epidural space } & \multicolumn{2}{|c|}{ Dorsal epidural space } \\
\hline & $1.5 \mathrm{ml}$ & $3.0 \mathrm{ml}$ & $1.5 \mathrm{ml}$ & $3.0 \mathrm{ml}$ \\
\hline SIVD & $59 / 82(71.9)^{*}$ & $67 / 82(81.7)^{*}$ & $30 / 82(36.6)$ & $35 / 82(42.7)$ \\
\hline IIVD & $73 / 82(89.0)$ & $78 / 82(95.1)$ & $47 / 82(57.3)$ & $50 / 82(61.0)$ \\
\hline
\end{tabular}

Values are number of patients (\%) with contrast spreading to the SIVD. SIVD: Supra-adjacent Intervertebral Disc, IIVD: Infra-adjacent Intervertebral Disc, RIVEI: retrograde interlaminar ventral epidural injection. $* \mathrm{P}<0.05 ; 1.5 \mathrm{ml}$ vs $3.0 \mathrm{ml}$ using the McNemar test.

Table 4. Mean Levels of Cephalad and Caudad Spread of Contrast from Site of RIVEI

\begin{tabular}{|c|c|c|c|c|}
\hline & \multicolumn{2}{|c|}{ Ventral epidural spread } & \multicolumn{2}{|c|}{ Dorsal epidural spread } \\
\hline & $1.5 \mathrm{ml}$ & $3.0 \mathrm{ml}$ & $1.5 \mathrm{ml}$ & $3.0 \mathrm{ml}$ \\
\hline Cephalad and caudad & $1.60 \pm 0.71^{*}$ & $2.21 \pm 0.93^{*}$ & $0.67 \pm 0.88^{\dagger}$ & $1.03 \pm 1.25^{\dagger}$ \\
\hline Cephalad only & $1.25 \pm 0.62^{\ddagger}$ & $1.66 \pm 0.79^{\ddagger}$ & $0.56 \pm 0.75^{\S}$ & $0.83 \pm 1.03^{\S}$ \\
\hline Caudad only & $0.34 \pm 0.36^{\|}$ & $0.55 \pm 0.42^{\S}$ & $0.10 \pm 0.22^{\pi}$ & $0.20 \pm 0.33^{\natural}$ \\
\hline
\end{tabular}

One level defined as in Fig. 2. RIVEI: retrograde interlaminar ventral epidural injection. ${ }^{*, t, \text {, }, \text {, } \|, ~}{ }^{\mathrm{P}}<0.05 ; 1.5 \mathrm{ml}$ vs $3.0 \mathrm{ml}$ using paired t test. 
superior to TFESI with respect to ventral contrast flow and effectiveness. We find their conclusion to be in error, however, as the authors in fact compared PIL with the superoposterior intervertebral foraminal placement of a transforaminal injection [1]. Furthermore, although the injection was performed at the most lateral part of the interlaminar space, the depth of spreading into the ventral epidural space anterior to the nerve root and the dural sac by this route may be inconsistent [3]; and in rare instances, the PIL technique may result in nerve injury [19]. The inferior foraminal and retrodiscal approach directly targets the origin of pain, the disc-nerve interface, but requires care to avoid disc puncture, as it is essentially a discographic needle path [12]. In one study using the LIVE approach [3], the catheter was placed correctly at the ventrolateral position of the nerve root in the gorge of the pedicle and the nerve root, but evaluation of the image in that publication did not clearly show nerve root filing.

During RIVEI, as described herein, the catheter tip placed into the dorsal epidural space through the Tuohy needle was advanced to the ventrocaudal aspect of the spinal nerve fascicles at the caudal level of the pedicle, and even to the rostral margin of the intervertebral disc. This was done because the catheter must pass through the axilla area between the main dural sac and the diverging nerve root sleeve, where fat is abundant [20]. Passage of the catheter is relevant to the hypotenuse of the Kambin triangle, and two studies $[7,8]$ have confirmed the safety of procedures performed within the triangle. Consequently, the relative safety of an endoscopic foraminotomy using a large bore trochar predicts the safety of RIVEI with respect to the nerve root and vascular injury.

The TFESI is expected to differ from alternative approaches [2-5] with respect to contrast spreading because of the distinctive localization of the needle tip. Patterns of contrast flow following fluoroscopically guided TF injections are well documented $[2,13,21]$; however, we found no studies that evaluated epidural contrast spreading patterns following the use of alternative approaches [2-5] to TF injection. This study was designed to evaluate these topics with fluoroscopically guided RIVEI.

An area commonly targeted in disc disorders is the ventral epidural space at the interface of the disc and the nerve root sleeve. However, pain generators in stenosis may differ from those involved in a central disc herniation [11]. To appropriately resolve inflammation and pain, and to prevent further recurrences, delivery of anti-inflammatory medications to all relevant target areas should be attempted [3].

Following injection of $3.0 \mathrm{ml}$ contrast, we observed ventral epidural spreading in all patients (Table 3). Manchikanti et al. [13] reported ventral epidural filling during $88 \%$ of TF procedures. Candido et al. [2] also reported ventral epidural filling during TFESI, with an overall rate of 75\% (21/28). With fluoroscopically guided lumbar TFESI, however, ventral epidural flow was observed in all patients [21]. As compared to previous studies $[2,13,21]$, we observed a higher rate of ventral epidural contrast filling during RIVEI. It is encouraging to note that ventral epidural filling was achieved in the majority of the patients, because this pattern corresponds to optimal delivery of injected materials (e.g., medication) to the target site at the ventral aspect of the lumbar nerve root sleeve and the dorsal aspect of disc herniation. Thus, our results imply that use of RIVEI may improve clinical outcomes as compared to TFESI. Such a conclusion, based on studies using different techniques with different groups of patients $[2,13,21]$, would be premature; however, a study designed to test this implication specifically may be justified.

In contrast to the observations of ventral epidural spreading in our study, dorsal epidural spreading was observed in only one-half of patients following a $3.0 \mathrm{ml}$ injection of contrast medium (Table 3). However, Botwin et al. [21] reported dorsal epidural contrast flow in $20 \%$ of patients in fluoroscopically guided lumbar TFESIs following a $2 \mathrm{ml}$ injection. In another study, dorsal epidural contrast spreading was observed in $9 \%$ of 239 procedures with TFESIs [13]. Although the rate of dorsal epidural spreading may be lower than that of ventral epidural spreading during RIVEI, the catheter tip position in RIVEI showed greater dorsal flow than the needle tip position in TFESI. Central stenosis usually involves a combination of dorsal and ventral obstructions of the spinal canal. Thus we venture to suggest that positioning the catheter tip by the RIVEI approach to improve dorsal epidural spreading may improve outcomes in central stenosis. Conceivably, in the presence of dorsal epidural disease or more generalized pathology such as multifactorial spinal stenosis, RIVEI may provide a more favorable placement.

During interlaminar epidural injection, the mean numbers of levels of contrast flow cephalad and caudad from the injection site were 1.28 and 0.88 , respectively [22], following injection of 5 $\mathrm{ml}$ of contrast medium. Contrast spreading during TFESI compares favorably with interlaminar injection. In a randomized prospective observational study, Candido et al. [2] assessed contrast flow patterns in the epidural space using the TF approach with continual fluoroscopic guidance. The mean grade of spread was 1.46 in the TF group with $5 \mathrm{ml}$ of contrast. In a prospective study of 20 consecutive patients receiving lumbar TFESI, Botwin et al. [21] found that the mean numbers of contrast flow levels cephalad and caudad from the injection site were $1.13 \pm 0.43$ and $0.6 \pm 0.21$, respectively, after a $2 \mathrm{ml}$ injection of contrast medium. To optimize the therapeutic benefit, the injection should direct the flow of medication to the spinal segments that correlate anatomically with the patient's clinical and radiographic presentation [11]. In principle, extending the flow from an injection to a greater number of vertebral levels should result in superior coverage of the site of pathology in the ventral epidural space. In this study, the mean numbers of levels of contrast flow cephalad 
and caudad from the injection site in the ventral epidural space were similar to numbers reported by Candido and Botwin [2,21]. Based on the contrast spreading observed in the ventral epidural space, the benefits of RIVEI may be comparable to those of TFESI.

In a study of 69 patients, Furman et al. [11] quantified contrast volumes in lumbar TFESI, aiming to optimize the benefits obtained through treatment by this procedure. To evaluate potential therapeutic coverage of the superior disc, the authors used SIVD as a specific anatomic landmark [11]. After injections of 1.5 and $2.8 \mathrm{ml}$ of contrast, $66.7 \%$ (38/60) and 95\% (57/60), respectively, of lumbar TFESI performed in that study showed spread to the SIVD corresponding to the level of injection. These evaluations assume that contrast spreads equally to the ventral and dorsal epidural spaces. An assessment of the imaging in that publication confirms equal levels of contrast spreading into the ventral and dorsal epidural spaces. Consequently, the injection volumes needed to cover the SIVD in the ventral and dorsal epidural spaces individually was not evaluated. Using $3 \mathrm{ml}$ injections of contrast, we found contrast spreading through the ventral epidural space to the SIVD in $81.7 \%$ of patients (Table 5). Our inclusion criteria were relatively broad, in that patients with disc herniation and spinal stenosis were enrolled. This provides a supplementary explanation for differences between our results and those of Furman et al. [11]. We found that $3.0 \mathrm{ml}$ of contrast reaches the inferior aspect of the inferior intervertebral disc in 95\% of injections (Table 5). However, in almost the same proportion of RIVEI performed in this study (81.7\%), a $3 \mathrm{ml}$ injection of contrast spread to cover both the SIVD and IIVD. This suggests that the delivery from one injection may be sufficient to treat both the superior and inferior levels, i.e., to treat two adjacent spinal segments [11]. As previously noted [11], a one-level, rather than a two-level injection may be especially favored for patients with a bleeding risk or for those with pathology extending across two levels.

Previous studies $[2,18,23,24]$ have evaluated outcome follow- ing TFESI in patients with radiating pain, predominantly unilateral, related to disease in a single spinal segment. Our study design may be criticized in that we did not similarly restrict inclusion so as to permit comparison of RIVEI data with those for TFESI. This study was also limited in the ability to maintain contrast injection rates. A single bolus volume of $0.5 \mathrm{ml}$ contrast was injected rapidly to confirm the intravascular spreading in several patients who were suspected of intravascular spreading. We expect that RIVEI would promote spreading along the nerve because of the greater pressure in rapid injection than in slow injection. However, as the succeeding volume of contrast was injected at the rate of $0.5-1.0 \mathrm{ml} / \mathrm{min}$, the influence of a single bolus injection of $0.5 \mathrm{ml}$ contrast on the extent of overall contrast spreading is not expected to be great. A further limitation of this study stems from potential differences in viscosity and epidural flow characteristics of anesthetic and steroid preparations as compared to the contrast. We performed the study assuming comparable flow rates for all agents injected by these techniques [25].

The principal finding of this study is that use of a retrograde interlaminar dorsal epidural approach in conjunction with ventral epidural catheterization may provide an effective method to deliver medication for radiating pain in the lower extremities. RIVEI may avoid certain risks related to the TF approach, particularly in the setting of foraminal abnormality. One intent of this study was to evaluate the ventral and dorsal epidural contrast spread using RIVEI; and based on these results, administration of a one-level RIVEI may be sufficient where a two-level injection would currently be used. Patients with a bleeding risk or with two-level central pathology may benefit in particular from this use of RIVEI. Our study does not present data of functional improvement or pain relief following RIVEI because we designed the study specifically to assess the spread of contrast during this procedure; however, observational and controlled studies of RIVEI in clinical applications may be justified.

\section{References}

1. Desai MJ, Shah B, Sayal PK. Epidural contrast flow patterns of transforaminal epidural steroid injections stratified by commonly used final needle-tip position. Pain Med 2011; 12: 864-70.

2. Candido KD, Raghavendra MS, Chinthagada M, Badiee S, Trepashko DW. A prospective evaluation of iodinated contrast flow patterns with fluoroscopically guided lumbar epidural steroid injections: the lateral parasagittal interlaminar epidural approach versus the transforaminal epidural approach. Anesth Analg 2008; 106: 638-44.

3. Choi YK, Barbella JD. Evaluation of epidurographic contrast patterns with fluoroscopic-guided lumbar interlaminar ventral epidural injection. Pain Pract 2009; 9: 275-81.

4. Jasper JF. Lumbar retrodiscal transforaminal injection. Pain Physician 2007; 10: 501-10.

5. Lew HL, Coelho P, Chou LH. Preganglionic approach to transforaminal epidural steroid injections. Am J Phys Med Rehabil 2004; $83: 378$.

6. Murthy NS, Maus TP, Behrns CL. Intraforaminal location of the great anterior radiculomedullary artery (artery of Adamkiewicz): a retrospective review. Pain Med 2010; 11: 1756-64. 
7. Kambin P. Arthroscopic microdiskectomy. Mt Sinai J Med 1991; 58: 159-64.

8. Kambin P, Savitz MH. Arthroscopic microdiscectomy: an alternative to open disc surgery. Mt Sinai J Med 2000; 67: 283-7.

9. Glaser SE, Shah RV. Root cause analysis of paraplegia following transforaminal epidural steroid injections: the 'unsafe' triangle. Pain Physician 2010; 13: 237-44.

10. Alo K, Yland MJ, Redko V, Feler C, Naumann C. Lumbar and sacral nerve root stimulation (NRS) in the treatment of chronic pain: a novel anatomic approach and neurostimulation technique. Neuromodulation 1999; 2: 23-31.

11. Furman MB, Mehta AR, Kim RE, Simon JI, Patel R, Lee TS, et al. Injectate volumes needed to reach specific landmarks in lumbar transforaminal epidural injections. PM R 2010; 2: 625-35.

12. Nagaro T, Yorozuya T, Kamei M, Kii N, Arai T, Abe S. Fluoroscopically guided epidural block in the thoracic and lumbar regions. Reg Anesth Pain Med 2006; 31: 409-16.

13. Manchikanti L, Cash KA, Pampati V, Damron KS, McManus CD. Evaluation of lumbar transforaminal epidural injections with needle placement and contrast flow patterns: a prospective, descriptive report. Pain Physician 2004; 7: 217-23.

14. Houten JK, Errico TJ. Paraplegia after lumbosacral nerve root block: report of three cases. Spine J 2002; 2: 70-5.

15. Kennedy DJ, Dreyfuss P, Aprill CN, Bogduk N. Paraplegia following image-guided transforaminal lumbar spine epidural steroid injection: two case reports. Pain Med 2009; 10: 1389-94.

16. Lyders EM, Morris PP. A case of spinal cord infarction following lumbar transforaminal epidural steroid injection: MR imaging and angiographic findings. AJNR Am J Neuroradiol 2009; 30: 1691-3.

17. Wybier M. Lumbar epidural and foraminal injections: update. J Radiol 2010; 91: 1079-85.

18. Park CH, Lee SH, Park HS. Lumbar retrodiscal versus post-ganglionic transforaminal epidural steroid injection for the treatment of lumbar intervertebral disc herniations. Pain Physician 2011; 14: 353-60.

19. Glaser SE, Shah RV, Finch PM. In response: are selective angiograms the answer for the high risk lumbar epidural steroid injection. Pain Physician 2010; 13: 397-9.

20. Hogan QH. Labat lecture: the primary sensory neuron: where it is, what it does, and why it matters. Reg Anesth Pain Med 2010; $35: 306-11$.

21. Botwin K, Natalicchio J, Brown LA. Epidurography contrast patterns with fluoroscopic guided lumbar transforaminal epidural injections: a prospective evaluation. Pain Physician 2004; 7: 211-5.

22. Botwin KP, Natalicchio J, Hanna A. Fluoroscopic guided lumbar interlaminar epidural injections: a prospective evaluation of epidurography contrast patterns and anatomical review of the epidural space. Pain Physician 2004; 7: 77-80.

23. Jeong HS, Lee JW, Kim SH, Myung JS, Kim JH, Kang HS. Effectiveness of transforaminal epidural steroid injection by using a preganglionic approach: a prospective randomized controlled study. Radiology 2007; 245: 584-90.

24. Paidin M, Hansen P, McFadden M, Kendall R. Contrast dispersal patterns as a predictor of clinical outcome with transforaminal epidural steroid injection for lumbar radiculopathy. PM R 2011; 3: 1022-7.

25. Furman MB, Lee TS, Mehta A, Simon JI, Cano WG. Contrast flow selectivity during transforaminal lumbosacral epidural steroid injections. Pain Physician 2008; 11: 855-61. 